\title{
Effects of Menstrual Cycle on Cardiac Autonomic Innervation As Assessed By Heart Rate Variability
}

\author{
Aylin Yildirir, M.D., Giray Kabakci, M.D., Ebru Akgul, M.D., \\ Lale Tokgozoglu, M.D., F.E.S.C., F.A.C.C., \\ and Ali Oto, M.D., F.E.S.C., F.A.C.C. \\ From the Hacettepe University Department of Cardiology, Ankara, Turkey
}

Objective: The aim of the study was to investigate the effects of menstrual cycle on cardiac autonomic function parameters in young healthy women by means of heart rate variability (HRV).

Methods: Forty-three nonobese regularly cycling women (age $29 \pm 6$, range 20-38) were enrolled. Recordings for HRV analysis were obtained during the two phases of the menstrual cycle when the estrogen and progesterone levels peaked (follicular phase $11 \pm 1$ days and luteal phase $21 \pm 1$ days from the start of bleeding). Power spectral analysis of HRV was performed to calculate the low frequency peak (LF, 0.04-0.15 Hz), high frequency peak (HF, 0.15-0.40 Hz), LF in normalized unit ( $\mathrm{LF} \mathrm{nU}$ ), $\mathrm{HF}$ in normalized unit (HF nU), and LF/HF ratio during the two phases of menstrual cycle.

Results: The heart rates, LF and HF, were similar in both phases $(P>0.05)$. A significant increase was noted in the LF NU in the luteal phase compared to follicular phase of the menstrual cycle (P= 0.014 ), whereas a tendency for increased HF NU was observed in the follicular phase (P $=0.053$ ). Furthermore, LF/HF ratio was significantly higher in the luteal phase compared to follicular phase $(2.1 \pm 1.5$ vs $1.6 \pm 0.9, \mathrm{P}=0.002)$, suggesting increased sympathetic activity in the luteal phase.

Conclusion: We concluded that regulation of autonomic tone is modified during menstrual cycle. The alteration in the balance of ovarian hormones might be responsible for these changes in the cardiac autonomic innervation.

A.N.E. 2002;7(1):60-63

heart rate variability; menstrual cycle; autonomic nervous system

A wide spectrum of biological functions is regulated by the cyclic changes in estrogen and progesterone levels during regular menstrual cycle. However little information is available concerning the possible interrelationships of these hormones and cardiovascular autonomic function.

Heart rate variability (HRV) measurement from electrocardiographic recordings has been shown to be useful to assess cardiac autonomic function. The frequency specific fluctuations in heart rate behavior and the sympathetic/parasympathetic integrity can be evaluated noninvasively with power spectrum analysis of HRV. ${ }^{1}$

There are a limited number of studies related to the effect(s) of menstrual cycle on cardiac auto- nomic function, as assessed by HRV or baroreflex sensitivity, with contradictory results. ${ }^{2-4}$ The aim of this study was to investigate the characteristics of cardiac autonomic function during the two different phases of menstrual cycle by HRV.

\section{METHODS}

\section{Study Population}

Young nonobese female health personnel with regular menses were enrolled into the study. All were free of any cardiac or neurological symptoms and had the history of regular ovulatory cycles for at least 24 months before inclusion. None were

Address for reprints: Aylin Yildirir, M.D., Simon Bolivar Cad. No:18/34, 6550 Cankaya Ankara/Turkey. Fax: 903124413553 ; E-mail: ayliny@ato.org.tr 
taking oral contraceptives, hormone replacement therapy, cardiovascular medications, or any other drugs. The HRV parameters of the subjects were investigated during the two phases of the menstrual cycle when the estrogen and progesterone levels peaked (follicular phase $11 \pm 1$ days and luteal phase $21 \pm 2$ days from the first day of bleeding).

The subjects included were questioned about symptoms of autonomic nervous system (ANS) dysfunction and underwent complete cardiac and neurologic examination. Baseline electrocardiograms (ECG) were obtained from all subjects and those with abnormal baseline ECG (including juvenile pattern) were excluded.

\section{Heart Rate Variability Analysis}

Recordings for HRV analysis were made with a PC-based high resolution ECG system (Kardiosis, Ankara, Turkey) after 30 minutes resting in a silent environment in supine position. Bipolar X deviation $\{0.5-340 \mathrm{~Hz}\}$ was recorded and sampled at a rate of 1000 samples per second and digitized using an 8-bit $\mathrm{A} / \mathrm{D}$ converter. Each recording lasted 8 minutes, and raw data were stored to disk for postprocessing. A segment of 5 minutes of the recording with no artefacts and premature depolar. izations was processed to provide the results of HRV analysis. The RR tachograms were extracted from the data. The detection of $R$ waves was done with a thresholding algorithm. The detected $R$ waves were visually confirmed and any undetected regular $\mathrm{R}$ wave was marked either manually or by interpolation. Similarly, any point which is not an $R$ wave but found as one, was unmarked manually. By this method, raw RR tachogram was extracted. Raw tachogram was then interpolated at 1-second intervals by linear interpolation. From the interpolated RR tachogram, power spectral densities were calculated by autoregressive modeling. Power spectrums for heart rate variations were calculated with autoregressive modeling. In power spectrums of RR intervals, three major peaks, one around 0.03 $\mathrm{Hz}$ (Very low frequency - VLF peak), one around $0.03-0.15 \mathrm{~Hz}$ (Low frequency - LF peak) and the other around $0.15-0.40 \mathrm{~Hz}$ (High frequency - HF peak) were seen. The peak around $<0.03 \mathrm{~Hz}$ was attributed to several physiologic variables and was filtered out from the tachograms before modeling. For spectral analysis, the following variables were calculated: (1) The low frequency component in absolute (LF) and normalized units (LF NU); (2) The high frequency component in absolute (HF) and normalized units (HF NU); (3) The ratio of the absolute low and high frequency component (LF) $\mathrm{HF}$ ). The absolute values of the results were in $\mathrm{ms}$ for the time domain and $\mathrm{ms}^{2}$ for power spectral analysis.

The power spectral analysis method of HRV from short-term recordings has been accepted as a reliable technique to evaluate the ANS functions ${ }^{5}$ and we used the same method in our previously published studies. ${ }^{6}$

\section{Statistical Analysis}

The Statistical Package for the Social Science (SPSS 9.1 version for Windows) was used for statistical analyses. Paired sample $t$-test was used to compare HRV parameters in both phases of menstrual cycle. Values were expressed as mean \pm SD. Statistical significance was set at $\mathrm{P}<0.05$.

\section{RESULTS}

Forty-three regularly cycling female health personnel were enrolled. The mean age and the body mass index of the subjects were $29 \pm 6$ years (range $20-38$ ) and $24 \pm 1 \mathrm{~kg} / \mathrm{m}^{2}$, respectively. Cardiac and neurological examination of the subjects did not reveal any abnormal findings.

The effects of menstrual cycle on various HRV parameters are given in Table 1 . The heart rates, $L F$, and HF were similar in both phases $(P>0.05)$.

Table 1. Spectral Analysis of Heart Rate Variability Parameters during the Two Phases of Menstrual Cycle

\begin{tabular}{lccc}
\hline & $\begin{array}{c}\text { Follicular } \\
\text { Phase } \\
\text { (n = 43) }\end{array}$ & $\begin{array}{c}\text { Luteal } \\
\text { Phase } \\
(\mathbf{n}=\mathbf{4 3})\end{array}$ & P value \\
\hline Heart rate & & & \\
$(\mathrm{beat} / \mathrm{min})$ & $76.4 \pm 12.5$ & $75.4 \pm 12.1$ & 0.516 \\
$\mathrm{LF}\left(\mathrm{m} / \mathrm{sn}^{2}\right)$ & $481.5 \pm 501.8$ & $559.1 \pm 539.5$ & 0.199 \\
$\mathrm{LF} \mathrm{nu}$ & $53.7 \pm 12.7$ & $58.2 \pm 14.2$ & 0.014 \\
$\mathrm{HF}\left(\mathrm{m} / \mathrm{sn}^{2}\right)$ & $384.9 \pm 480.8$ & $395.6 \pm 3511.8$ & 0.842 \\
$\mathrm{HF} \mathrm{nu}$ & $39.7 \pm 12.4$ & $36.6 \pm 13.9$ & 0.053 \\
$\mathrm{LF} / \mathrm{HF}$ & $1.6 \pm 0.9$ & $2.1 \pm 1.5$ & 0.002 \\
\hline
\end{tabular}

$\mathrm{HF}=$ high frequency; $\mathrm{HF} \mathrm{nu}=$ high frequency in normalized unit; $L F=$ low frequency; $L F$ nu = low frequency in normalized unit; $L F / H F=$ low frequency/high frequency ratio. 
A significant increase was noted in the LF NU in the luteal phase compared to follicular phase of the menstrual cycle $(\mathrm{P}=0.014)$, whereas, a tendency for increased HF NU was observed in the follicular phase $(\mathrm{P}=0.053)$. Furthermore, LF/HF ratio was significantly higher in the luteal phase compared to follicular phase $(2.1 \pm 1.5$ vs $1.6 \pm 0.9, \mathrm{P}=0.002\}$, indicating an increased sympathetic activity in the luteal phase.

\section{DISCUSSION}

The possible role of sex hormones on the ANS functions during the two phases of menstrual cycle has been suggested by Goldstein et al. ${ }^{7}$ who reported increased plasma catecholamine levels in the luteal phase of the menstrual cycle compared to follicular phase, despite the similarities in urinary catecholamine levels.

The first clinical study supporting this hypothesis has been reported by Sato et al., ${ }^{2}$ who investigated the fluctuations of ANS activities during the menstrual cycle in 20 college students. In this study, cardiovascular reactivity to mental challenge was tested with power spectral analysis of HRV in both follicular and luteal phases across two menstrual cycles.

Their results indicated increased LF component and decreased HF component of HRV during the luteal phase. The $\mathrm{LF} / \mathrm{HF}$ ratio was also significantly greater in the luteal phase than in the follicular phase. These findings are consistent with our results; however we evaluated HRV after 20 minutes of resting in supine position rather than a response to mental challenge.

Similar results have been reported by Guasti et al. ${ }^{3}$ who evaluated the cardiac autonomic function by power spectral analysis of HRV and baroreflex sensitivity on a relatively smaller study population ( $\mathrm{n}=13$ ) with regular menses. Their results indicated a significantly higher $\mathrm{LF} / \mathrm{HF}$ ratio in the presence of increased LF NU and reduced HF NU in the luteal phase of the cycle, indicating a relatively higher sympathetic activity during this phase. However, the baroreflex sensitivity did not significantly differ in the two phases.

In contrast to the above mentioned two studies and our study, an increased parasympathetic activity during the luteal phase has also been reported. Fuenmayor et al. ${ }^{4}$ investigated the cardiac auto- nomic system balance variations during two different stages of menstrual cycle by means of Valsalva maneuver in 20 women with regular menses. The Valsalva index (i.e., the ratio between the longest $R-R$ interval after releasing strain and the shortest $R-R$ interval during strain release) was used in that study to assess heart rate response on ECG as an indicator of cardiac autonomic balance. They reported significantly higher Valsalva index during the luteal phase compared to follicular phase, suggesting an increase in parasympathetic activity during the luteal phase of the menstrual cycle. However, this study was limited in methodology since it investigated the Valsalva index, which was a crude method for the evaluation of ANS functions rather than computer based power spectral analysis of HRV.

Our results are consistent with the results of Sato et al. ${ }^{2}$ and Guasti et al. ${ }^{3}$ by indicating increased sympathetic /parasympathetic ratio in the luteal phase of the menstrual cycle compared to follicular phase. Furthermore, to the best of our knowledge, our study population included the highest number of women reported so far on the effects of menstrual cycle on cardiac innervation.

Several mechanism(s) may be responsible for the increased sympathetic/parasympathetic ratio in the luteal phase. The presence of nuclear and cytosolic receptors for sex hormones within the cardiovascular system and its neuroregulatory tissues ${ }^{8}$ suggests the idea that sex hormones could exert direct and indirect effects on the cardiovascular system by these receptors and may regulate gene expression. However, the exact mechanism responsible for the sex hormone related change in the cardiac autonomic modulation is unclear.

We concluded that regulation of autonomic tone is modified during menstrual cycle. The alteration in the balance of ovarian hormones might be responsible for these changes.

\section{REFERENCES}

1. Fallen EL, Kamath MV, Ghista DN. Power spectrum of heart rate variability: A noninvasive test of integrated neurocardiac function. Clin Invest Med 1988;11:331-340.

2. Sato N, Miyake S, Akatsu J, et al. Power spectral anal$y$ sis of heart rate variability in healthy young women during the normal menstrual cycle. Psychosom Med 1995;57:331-335.

3. Guasti I, Grimoldi P, Mainardi LT, et al. Autonomic function and baroreflex sensitivity during a normal ovulatory cycle. Acta Cardiol 1999;54:209-213. 
4. Fuenmayor AJ, Ramirez L, Fuenmayor AM. Left ventricular function and autonomic nervous system balance during two different stages of the menstrual cycle. Intern J Cardiol $2000 ; 72: 243-246$.

5. Task Force of the European Society of Cardiology and the North American Society of Pacing and Electrophysiology. Heart rate variability: Standards of measurement, physiological interpretation and clinical use. Circulation 1996;93: 1043-1065.

6. Tokgozoglu SL, Batur MK, Topcuoglu MA, et al. Effects of stroke localization on cardiac autonomic balance and sudden death. Stroke 1999;30:1307-1311.

7. Goldstein DS, Levinson P, Keiser HR. Plasma and urinary catecholamines during the human ovulatory cycle. J Obstet Gynecol 1983;146:824-829.

8. Stumpf WE. Steroid hormones and the cardiovascular system: Direct actions of estradiol, progesterone, testosterone, gluco- and mineralocorticoids and soltriol (vitamin D) on central nervous regulatory and peripheral tissues. Experientia 1990:46:13-25. 